\title{
Role of Phytopathogenic Fungi in Forest Plant Breeding- Development of DNA-Based Quick Tests for Quality Assurance in Forest Plant Production ${ }^{+}$
}

\author{
Kristin Morgenstern ${ }^{1, *}$, Jens-Ulrich Polster ${ }^{1}$, Birgit Reiche ${ }^{1}$, Patrick Schützel ${ }^{2}$, Imke Hutter ${ }^{3}$ and Doris Krabel ${ }^{1}$ \\ 1 Faculty of Environmental Sciences, Institute of Forest Botany and Forest Zoology, Working Group \\ "Molecular Physiology of Woody Plants", Technische Universität Dresden, Pienner Straße 7, \\ 01737 Tharandt, Germany; jens-ulrich.polster@tu-dresden.de (J.-U.P.); birgit.reiche@tu-dresden.de (B.R.); \\ doris.krabel@tu-dresden.de (D.K.) \\ 2 Biomasse Schraden e. V., Straße zum Stützpunkt 15, 04932 Großthiemig, Germany; \\ patrickschuetzel@biomasse-schraden.de \\ 3 Institut für Pflanzenkultur e. K., Solkau 2, 29465 Schnega, Germany; hutter@pflanzenkultur.de \\ * Correspondence: Kristin.Morgenstern@tu-dresden.de; Tel.: +49-351-463-31836 \\ † Presented at the 1st International Electronic Conference on Forests - Forests for a Better Future: Sustainability, \\ Innovation, Interdisciplinarity, 24-27 June 2020; Available online: https://iecf2020.sciforum.net.
}

Citation: Morgenstern, K.;

Polster, J.-U.; Reiche, B.; Schützel, P.; Hutter, I.; Krabel, D. Role of

Phytopathogenic Fungi in Forest Plant Breeding-Development of DNA-Based Quick Tests for Quality Assurance in Forest Plant Production. Environ. Sci. Proc. 2021, 3, 96. https://doi.org/10.3390/ IECF2020-07898

Academic Editors: Angela Lo Monaco, Cate Macinnis-Ng and Om P. Rajora

Published: 11 November 2020

Publisher's Note: MDPI stays neutral with regard to jurisdictional claims in published maps and institutional affiliations.

Copyright: (C) 2020 by the authors. Licensee MDPI, Basel, Switzerland. This article is an open access article distributed under the terms and conditions of the Creative Commons Attribution (CC BY) license (http://creativecommons.org/licenses /by/4.0/).
Abstract: The production of healthy seed and plant material is a fundamental prerequisite for the establishment of ecologically stable and economically productive forest stands. As in the past, forest plant production is nevertheless threatened by harmful biotic factors, with new, invasive species playing an increasingly significant role as a result of climate change and globalization. DNA-based methods have significantly accelerated the detection of plant pathogens, but are still timeconsuming, costly, and require extensive equipment. Loop-mediated isothermal amplification (LAMP) is an efficient and cost-effective alternative to conventional polymerase chain reaction (PCR). The LAMP reaction is performed as a one-step assay at constant temperature and can be evaluated visually. The ongoing project "TreeLAMP" has led to the establishment of an LAMP assay for Rhabdocline pseudotsugae, one of the most important needle pathogens of Douglas fir. To date, 32 sets of LAMP primers have been derived from the internal transcribed spacer (ITS) region of ribosomal DNA (rDNA) and tested. The results show clear differences between the primer sets both in terms of reaction rate and concentration of amplified products. Following extensive work targeting the optimization of the LAMP reaction, a method is now available that enables the reliable detection of $R$. pseudotsugae at a constant temperature $\left(65^{\circ} \mathrm{C}\right)$ and after a reaction time of $1.5 \mathrm{~h}$. The detection limit is currently $0.02 \mathrm{pg} / \mu \mathrm{L}$ of $R$. pseudotsugae DNA. The current focus of the project is the optimization of DNA extraction. In addition to conventional DNA kits, methods that are specially adapted to the detection procedure are also under investigation. These will allow DNA extraction to occur faster and without any great technical effort.

Keywords: isothermal amplification; LAMP; Rhabdocline pseudotsugae; Douglas fir

\section{Introduction}

The production of healthy seed and plant material is a fundamental prerequisite for the establishment of ecologically stable and economically productive forest stands. As in the past, forest plant production is nevertheless threatened by harmful biotic factors, with new, invasive species playing an increasingly significant role as a result of climate change and globalization. From a forestry perspective, it is important that plant loss is reduced effectively and at a minimum of effort and cost. The fields of plant protection and plant breeding have a significant role to play in this regard, but novel strategies are necessary to meet the increasingly demanding requirements. The detection and identification of 
plant pathogens is one of the key prerequisites for the development of treatment concepts and the implementation of plant protection measures. Early detection in seed harvesting stands, seeds, and seedlings is of particular interest in this regard, and demands the development of efficient, practical diagnostic methods.

Methods based on the polymerase chain reaction (PCR) are generally characterized by a high level of sensitivity, specificity, and reliability. They are therefore already routinely used to identify pathogens in a forestry context. Examples include PCR-based methods for the detection of pathogens responsible for Dothistroma needle blight [1,2], various species of the genus Fusarium [3,4], Phytophthora [5,6], and Rhabdocline [7], as well as Sphaeropsis sapinea (Fr.) Dyko \& B. Sutton [8], Hymenoscyphus pseudoalbidus V. Queloz et al. [9], and many other pathogens. Such methods are nevertheless time-consuming and require a level of investment in equipment that plant production companies are generally unable to afford. Loop-mediated isothermal amplification (LAMP) represents a sensitive and, above all, simple alternative to conventional PCR. The method was developed by Notomi et al. [10] for the detection of hepatitis B viruses. The LAMP amplification involves the use of four to six primers that bind at six to eight regions of the target DNA. The synthesis of the new DNA strand occurs with the aid of a thermostable DNA polymerase characterized by a high level of strand displacement activity, thus enabling amplification to occur at constant temperature in a simple block heater or water bath. Another advantage is the simplicity of the results evaluation process. LAMP amplified products can be stained using fluorescent dyes and are therefore visible to the naked eye immediately after the reaction. The method is therefore particularly suitable for in situ analysis and laboratories that lack PCR equipment. A growing number of LAMP protocols for the detection of pathogens affecting herbaceous and woody plants have been established in recent years. To give an example, OptiGene Ltd. (Horsham, UK) offers an LAMP kit for the detection of H. pseudoalbidus [11]. Amongst others, LAMP protocols have also been published for Fusarium graminearum Schwabe [12], Botrytis cinerea Pers. [13], Heterobasidion irregulare Garbelotto \& Otrosina [14], Xylella fastidiosa Wells et al., Ceratocystis platani Engelbrecht \& Harrington, and Phytophthora ramorum Werres et al. [15].

This paper presents the development of an LAMP-based quick test in combination with LAMP-optimized DNA extraction as a means of detecting Rhabdocline pseudotsugae Syd. in plant material from Douglas firs. The objective is to establish a robust detection method for practical forestry scenarios that not only enables plant production businesses to examine plant material quickly and easily, but also enhances quality management processes in the production and distribution of "source-identified" plant material over the long term.

\section{Materials and Methods}

\subsection{Fungal Material, Plant Material}

The establishment of the new LAMP method involved the use of a synthetic Rhabdocline fragment as a positive control. The templates for the synthetic fragment were a consensus sequence from the internal transcribed spacer (ITS) regions of the ribosomal DNA (rDNA) of R. pseudotsugae (the regions were available in the Genome Database maintained by the National Center for Biotechnology Information (NCBI)) as well as proprietary sequence data on ITS regions gathered within the context of previous studies. The Rhabdocline fragment was synthesized by a DNA service provider (Eurofins Genomics GmbH, Ebersberg, Germany).

Branch material with visible fruiting bodies of R. pseudotsugae and branch material with no visible signs of infection were collected from a roughly 25-year-old stand of Douglas firs in Tharandter Wald in Saxony $\left(50^{\circ} 58^{\prime} \mathrm{N}, 13^{\circ} 28^{\prime} \mathrm{E}\right)$ in 2018 and 2019 before being stored at $-40{ }^{\circ} \mathrm{C}$ for further examination. Douglas fir seedlings from four German designated regions of origin for reproductive forest plant material were cultivated by project partner Biomasse Schraden e. V. (Großthiemig, Germany) in 2018 and also made 
available for the purpose of the study. Table 1 summarizes the origin (region and forest stand), year of ripeness, and germination capacity of the seeds used:

Table 1. Seeds used for the cultivation of seedlings by Biomasse Schraden e. V., including data on region of origin, forest stand, year of ripeness, and germination capacity.

\begin{tabular}{cccc}
\hline Region of Origin & Forest Stand & Year of Ripeness Germination Capacity \\
\hline North-East German lowlands, excluding Schles- & FoA Billenhagen & 2011 & not specified \\
wig-Holstein (85302) & Rev. Altheide & & $81.0 \%$ \\
Central and East German lowlands and hills (85303) & Mittweida & 2016 & $87.0 \%$ \\
West and South German hills, mountains and alpine & Stadt Dillenburg & 2016 & $81.0 \%$ \\
terrain (85305) & Grillenburg & 2016 & \\
South-East German hills and mountains (85306) &
\end{tabular}

The specificity of the LAMP reaction was tested using isolates of endophytic fungi supplied by project partner Institut für Pflanzenkultur e. K. (IFP; Schnega, Germany). The isolates were cultivated from surface-disinfected Douglas fir needles. The plant material was collected from the aforementioned Douglas fir stand in Tharandter Wald in spring 2018. DNA was extracted from the fungal isolates using an innuPREP Plant DNA Kit, Protocol 1 (Analytik Jena AG, Jena, Germany) and stored at $-25^{\circ} \mathrm{C}$. Table 2 summarizes the samples used. DNA taken from an environmental sample colonized with arbuscular mycorrhizal fungi (AMF) from a greenhouse trial at project partner IFP was also made available (see Table 2). The fungal species present in the AMF sample were determined using targeted amplified product sequencing of the ITS region of the rDNA with the aid of nanopore sequencing (Oxford Nanopore Technologies Ltd., Oxford, UK) followed by taxonomical classification of the sequences using the Kaiju program [16].

Table 2. List of fungal isolates from Douglas fir needles (nos. 1-15) and a greenhouse sample colonized with arbuscular mycorrhizal fungi (no. 16; supplied by Institut für Pflanzenkultur e. K.) used to test the specificity of the loop-mediated isothermal amplification (LAMP) primers, including data on the number of isolates tested per species.

\begin{tabular}{ccc}
\hline No. & Species & $\begin{array}{c}\text { Number of } \\
\text { Isolates Tested }\end{array}$ \\
\hline 1 & Anthostomella pinea Crous & 1 \\
2 & Cladosporium sp. & 1 \\
3 & Desmazierella acicola Lib. & 1 \\
4 & Diaporthe sp. & 2 \\
5 & Eurotiomycetes & 1 \\
6 & Fungal sp. & 1 \\
7 & Lophiostoma corticola (Fuckel) E.C.Y. Liew, Aptroot \& K.D. & 1 \\
8 & Hyde & 1 \\
9 & Nemania serpens (Pers.) Gray & 3 \\
10 & Nothophaeocryptopus gaeumannii (T. Rohde) Videira, C. & 1 \\
11 & Pestalotiopsis sp. & 1 \\
12 & Peziza arvernensis Boud. & 1
\end{tabular}




\subsection{LAMP Primer Design}

A set of LAMP primers consists of a basic set comprising an inner primer pair (FIP/BIP) and an outer primer pair (F3/B3). Reaction rate can be improved with the aid of additional loop primers (LF/LB). In the study at hand, suitable LAMP primers were selected using the PrimerExplorer V5 (Eiken Chemical Co., Ltd.; Tokyo, Japan). It was possible to use the Rhabdocline-specific consensus sequence of the ITS region to identify a large number of potential primer binding sites. Five basic sets with binding sites between the $5.8 \mathrm{~S}$ and $28 \mathrm{~S}$ rRNA genes were selected alongside three basic sets that bind in the ITS1 and ITS2 region. Wherever possible, additional loop primers (LF/LB) were determined and a TTTT spacer variation in the FIP and BIP primer tested for each basic set. The specificity of the binding sites was tested using BLAST analysis.

\subsection{Establishment of the LAMP Assay}

A total of 32 LAMP primer combinations were compared in terms of their reaction rate and yield, as well as the reproducibility and specificity of the amplification. Tests were carried out using the WarmStart LAMP Kit, WarmStart Colorimetric LAMP 2x Master Mix and Bst 3.0 DNA polymerase from New England Biolabs GmbH (Frankfurt am Main) as well as the GspSSD Isothermal Mastermix and the GspSSD 2.0 Isothermal Mastermix from OptiGene Ltd. (supplied by Amplex Diagnostics $\mathrm{GmbH}$, Gießen, Germany). All work carried out in connection with the establishment of the LAMP assay was conducted using a qTower ${ }^{3}$ real-time PCR cycler (Analytik Jena AG).

Project partner Biomasse Schraden e. V. provided 50 Douglas fir seedlings per region of seed origin for the examination of the sensitivity of the LAMP reaction. DNA was extracted using the DNeasy Plant Mini Kit (Qiagen GmbH, Hilden, Germany) in accordance with the manufacturer's instructions. All samples were first tested for infection with R. pseudotsugae with the aid of nested PCR. This involved an initial PCR using universal primers ITS1F/ITS4 $[17,18]$ followed by the nesting of the PCR products with R. pseudotsugae-specific primer pair RPP1/RPP4 [7] in a second PCR. A detailed description of the PCR parameters is provided in Morgenstern et al. [19]. Fragment length of the final PCR product was measured using a Fragment Analyzer ${ }^{\mathrm{TM}}$ (Agilent Technologies Deutschland $\mathrm{GmbH}$, Waldbronn, Germany). The results were evaluated using ProSize 3.0 software (Agilent Technologies Deutschland $\mathrm{GmbH}$ ).

The successful establishment of the LAMP assay was followed by the retesting of the DNA samples from the same seedlings for infection with R. pseudotsugae. The LAMP reaction mixture contained 15.0 $\mu \mathrm{L}$ of GspSSD Isothermal Master Mix (OptiGene Ltd.), 4.0 $\mu \mathrm{L}$ of RNase-free water (Qiagen $\mathrm{GmbH}$ ), $5.0 \mu \mathrm{L}$ of LAMP Primer Mix (Eurofins Genomics GmbH; FIP/BIP: $4.0 \mu \mathrm{M}$ each; F3/B3: $1.0 \mu \mathrm{M}$ each; LF/LB: $2.0 \mu \mathrm{M}$ each), and $1.0 \mu \mathrm{L}$ of the DNA template. The LAMP mixture was incubated for $1.5 \mathrm{~h}$ at $65^{\circ} \mathrm{C}$ in a qTower ${ }^{3}$ real-time PCR cycler (Analytik Jena AG).

\subsection{Optimization of DNA Extraction}

In the first step, the influence of Douglas fir DNA on the LAMP reaction was examined. This involved the mixing of a specific amount of R. pseudotsugae DNA with various amounts of Douglas fir DNA and the evaluation of the rate and yield of the LAMP reaction. A seedling from the Dillenburg stand that had been tested with $R$. pseudotsugaespecific primers RPP1/RPP4 and found to be free of infection was selected as the Douglas 
fir sample. A sample with a specific concentration of $R$. pseudotsugae was obtained by dissecting fruiting bodies from Douglas fir needles collected in Tharandter Wald and extracting DNA using the innuPREP Plant DNA Kit, Protocol 1 (Analytik Jena AG). The concentration of the fungal DNA was increased by means of standard PCR using primer pair ITS1F/ITS4, with the amplified ITS fragment subsequently diluted to $0.2 \mathrm{ng} / \mu \mathrm{L}$.

DNA extraction methods suitable for use in combination with LAMP amplification were researched from a variety of aspects. On the one hand, the methods tested included conventional DNA kits recommended by a variety of manufacturers for either the extraction of fungal DNA (DNeasy Plant Mini Kit (Qiagen GmbH); NucleoSpin ${ }^{\circledast}$ Plant II (Macherey-Nagel GmbH \& Co. KG, Düren); innuPREP Plant DNA Kit (Analytik Jena $\mathrm{AG})$ ) or the extraction of DNA from difficult environmental samples (E.Z.N.A. ${ }^{\circledR}$ Soil DNA Kit (Omega Bio-tek, Inc., distributed by VWR International GmbH, Darmstadt, Germany); innuPREP TCM DNA Extraction Kit; innuPREP Stool DNA Kit (Analytik Jena AG)). All tests were successfully used to extract DNA from Douglas fir needles featuring fresh fruiting bodies of R. pseudotsugae. The primary objective was to extract fungal DNA that was as pure as possible and free from inhibitors.

On the other hand, the work carried out targeted the identification of methods that facilitate quick DNA extraction at a minimum of technical effort. DNA extraction was tested with the aid of FTA Indicating Micro Cards (Whatman, part of GE Healthcare; distributed by VWR International $\mathrm{GmbH}$ ), the Dilution Buffer from the Phire Plant Direct PCR Kit (Fisher Scientific GmbH, Schwerte, Germany), and the Plant Material Lysis Kit (OptiGene Ltd.). The needle material used came from infected Douglas firs with visible fruiting bodies as well as needles without visible fruiting bodies.

\section{Results}

\subsection{Establishment of the LAMP Assay}

A total of 32 sets of LAMP primers (eight basic sets with variations) were initially tested using the WarmStart LAMP Kit (New England Biolabs GmbH). Five primer sets did not return a result. Amplification was essentially observed in all of the remaining 27 sets. They nevertheless exhibited significant differences in terms of both reaction rate and concentration of amplified products. Primer sets that featured loop primers as well as TTTT spacers in their FIP and BIP primers were generally found to achieve better amplification results than those that did not. As a basic principle, primer combinations located between $5.8 \mathrm{~S}$ and $28 \mathrm{~S}$ rRNA in the ITS2 region achieved shorter reaction times than sets with binding sites in the ITS1 and ITS2 regions.

A relatively long reaction time of four hours was selected for initial primer testing in order to determine if and when each individual set of primers yields amplified products. This nevertheless led to an elevated number of non-specific amplifications, though it is to be noted that they were significantly different from those involving $R$. pseudotsugae in terms of yield and reaction time. This notwithstanding, attempts were made to optimize the stability and reaction rate of the amplification. A variety of different polymerases, primer concentrations, and primer ratios were tested in the reaction mixture. The type of polymerase used was found to have a substantial influence on the stability of the LAMP reaction. The WarmStart LAMP Kit and WarmStart Colorimetric LAMP 2x Master Mix (both from New England Biolabs $\mathrm{GmbH}$ ) exhibited a distinct tendency towards nonspecific amplifications after a reaction time of two or more hours. The Bst 3.0 DNA polymerase (also from New England Biolabs $\mathrm{GmbH}$ ) was not found to lead to an improvement. Polymerases from OptiGene Ltd. achieved the best results in terms of the stability of the LAMP reaction, whereby the GspSSD Isothermal Mastermix exhibited a far lower tendency towards non-specific amplification after a reaction time of two or more hours than the GspSSD2.0 Isothermal Mastermix. Significant differences between individual polymerases in terms of reaction rate were nevertheless not observed. Reaction rate is primarily influenced by the concentration of the template DNA. Figure 1 shows the 
amplification curves for the LAMP reaction at concentrations of between $200 \mathrm{pg} / \mu \mathrm{L}$ and $0.02 \mathrm{pg} / \mu \mathrm{L}$ of Rhabdocline DNA. As a basic principle it is possible to detect $R$. pseudotsugae up to a detection limit of $0.02 \mathrm{pg} / \mu \mathrm{L}$, yet as DNA concentration decreases, the cycle threshold (Ct) value shifts from 15.2 cycles at $200 \mathrm{pg} / \mu \mathrm{L}$ to 35.4 cycles at $0.02 \mathrm{pg} / \mu \mathrm{L}$. The $\mathrm{Ct}$ value denotes the measuring cycle at which fluorescence rises exponentially from the background and therefore describes the rate of the reaction. A single cycle corresponds with a reaction time of two minutes. A decrease in the amount of template DNA is therefore accompanied by a decrease in reaction rate. Although increases in primer concentration and changes in the mixing ratio between individual LAMP primers were only found to result in a negligible increase in reaction rate, they nevertheless led to an increase in the risk of non-specific amplification. Further attempts to optimize the reaction rate were therefore neglected in favor of reaction stability.

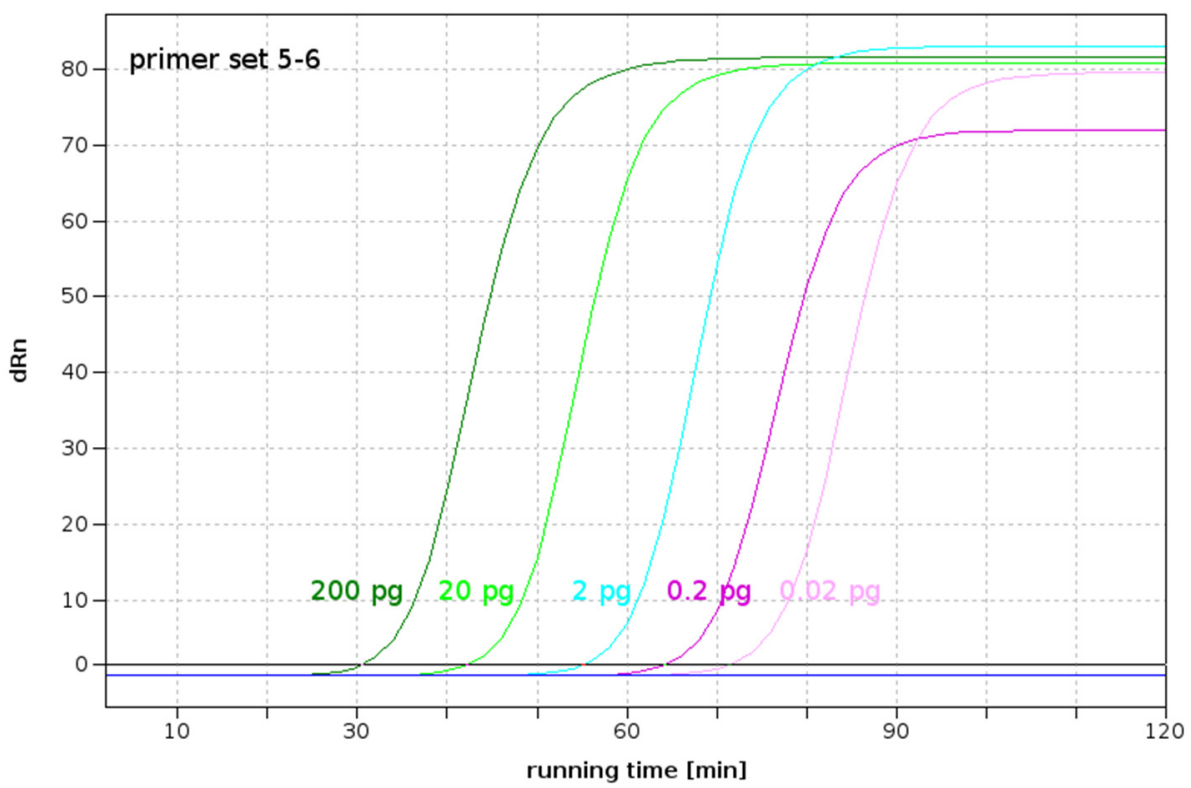

Figure 1. Amplification curves generated by the $\mathrm{qTower}^{3}$ real-time PCR cycler (GspSSD Isothermal Mastermix; primer set 5-6; incubation time: $2 \mathrm{~h}$; 1 cycle $=2 \mathrm{~min}$ of running time): Comparison of reaction rate and yield with various concentrations of the synthetic Rhabdocline fragment (200 $\mathrm{pg} / \mu \mathrm{L}-0.02 \mathrm{pg} / \mu \mathrm{L})$.

It was observed that the use of primer set (set 5-6) in combination with GspSSD Isothermal Mastermix yields satisfactory, reproducible results after a reaction time of 1.5 $\mathrm{h}$ and with a detection limit of $0.02 \mathrm{pg} / \mu \mathrm{L}$ of $R$. pseudotsugae DNA. The sensitivity of the LAMP reaction was also tested using mixed samples containing $20 \mathrm{ng} / \mu \mathrm{L}$ of Douglas fir DNA (taken from a seedling from the Dillenburg stand) and between $200 \mathrm{pg} / \mu \mathrm{L}$ and 0.02 $\mathrm{pg} / \mu \mathrm{L}$ of $R$. pseudotsugae DNA. Differences in LAMP reaction sensitivity between pure fungal sample and mixed samples containing both fungal and Douglas fir DNA were not observed.

The specificity of the LAMP assay was tested using 15 different fungal isolates as well as the AMF sample. In the case of the fungal isolates (see Table 2, nos. 1-15), an initial test using primer set $5-6$ led to the observation of positive signals at $C t$ values of over 40 cycles in one of four $R$. parkeri isolates and in the P. macrostoma. The LAMP reaction was repeated with all four $R$. parkeri isolates, none of which returned an amplification product. Results were initially reproducible in the case of $P$. macrostoma. Amplification only failed to be observed after the repeated extraction of DNA from the P. macrostoma isolate. None of the other isolates returned a result after a reaction time of $1.5 \mathrm{~h}$. In the case of the AMF sample, it was possible to taxonomically classify 1.46 million fungal sequences further to quality 
control. A total of $50.3 \%$ of sequences could not be classified, with another $4.6 \%$ of sequences only classifiable to a higher taxonomic level. A total of 174 fungal species were identified. Rhabdocline pseudotsugae was not detected. BLAST analysis of the LAMP primers against the sequences in the AMF sample did not identify any primer binding sites that would facilitate an LAMP reaction. The LAMP reaction therefore did not result in the amplification of the AMF sample.

\subsection{Comparison between the LAMP Assay and Polymerase Chain Reaction (PCR)}

A total of 50 seedlings per region of seed origin (20 complete seedlings and 30 seedlings separated into needles, stems, and roots) were tested for infection with $R$. pseudotsugae. The results of this screening process are summarized in Table 3. Nested PCR with fungus-specific primers led to the detection of $R$. pseudotsugae in samples from all four regions of seed origin. The rate of infection in complete plants ranged between $15 \%$ in seedlings from Grillenburg and 30\% in seedlings from Dillenburg. As expected, $R$. pseudotsugae was most frequently detected in needles (24.5\%), with $18.3 \%$ of stems and $13.5 \%$ of roots also testing positive. The ITS product used for nested PCR was retained for the repetition of the screening process using LAMP. Rhabdocline pseudotsugae was again detected in plant material from all four regions of seed origin and in needles, stems, and roots. The proportion of positive tests was nevertheless significantly higher, with infection rates among complete seedlings found to range between 35\% (Grillenburg) and 55\% (Billenhagen). Overall, the newly-established LAMP assay identified a 21\% higher rate of infection across all samples tested than nested PCR, with an identical DNA template used in both cases.

Table 3. R. pseudotsugae screening: Comparison between results achieved using PCR and the LAMP reaction; figures describe the relative proportion (\%) of samples (complete plants, needles, stems, and roots) that tested positive for R. pseudotsugae per region of seed origin.

\begin{tabular}{ccccccccc}
\hline Origin & \multicolumn{2}{c}{ Complete } & \multicolumn{2}{c}{ Needles } & \multicolumn{2}{c}{ Stems } & \multicolumn{2}{c}{ Roots } \\
& PCR & LAMP & PCR & LAMP & PCR & LAMP & PCR & LAMP \\
\hline Billenhagen & $20 \%$ & $55 \%$ & $27 \%$ & $63 \%$ & $13 \%$ & $50 \%$ & $0 \%$ & $30 \%$ \\
Mittweida & $20 \%$ & $40 \%$ & $30 \%$ & $43 \%$ & $30 \%$ & $43 \%$ & $20 \%$ & $50 \%$ \\
Dillenburg & $30 \%$ & $40 \%$ & $18 \%$ & $36 \%$ & $7 \%$ & $23 \%$ & $17 \%$ & $45 \%$ \\
Grillenburg & $15 \%$ & $35 \%$ & $23 \%$ & $23 \%$ & $23 \%$ & $37 \%$ & $17 \%$ & $33 \%$ \\
\hline
\end{tabular}

\subsection{Optimization of DNA Extraction}

The influence of Douglas fir DNA on the sensitivity of the LAMP reaction was investigated by testing samples containing a constant amount of $R$. pseudotsugae DNA $(0.2$ $\mathrm{ng} / \mu \mathrm{L}$ ) mixed with various concentrations of Douglas fir DNA (1 ng/ $\mu \mathrm{L}$ to $60 \mathrm{ng} / \mu \mathrm{L})$. Samples containing pure R. pseudotsugae DNA $(0.2 \mathrm{ng} / \mu \mathrm{L})$ were also tested for the purpose of comparison. Figure 2 shows the $\mathrm{Ct}$ value and average fluorescence of the LAMP reaction as a factor of increasing concentration of Douglas fir DNA. Ct values fluctuated between 8.02 cycles ( $5 \mathrm{ng} / \mu \mathrm{L}$ of Douglas fir DNA) and 8.68 cycles $(10 \mathrm{ng} / \mu \mathrm{L}$ of Douglas fir DNA) up to a concentration of $10 \mathrm{ng} / \mu \mathrm{L}$. A slight increase in Ct values to 11.30 cycles (60 $\mathrm{ng} / \mu \mathrm{L}$ of Douglas fir DNA) was only observed at a concentration of $20 \mathrm{ng} / \mu \mathrm{L}$ and above. Douglas fir DNA, therefore, appears to have no significant influence on reaction rate.

Douglas fir DNA does, on the other hand, have a distinct influence on the amount of amplified LAMP products. This is demonstrated by the significant decrease in fluorescence intensity-and with it, the amount of amplified products-starting at a concentration of $10 \mathrm{ng} / \mu \mathrm{L}$ of Douglas fir DNA, with fluorescence values of just 7,239 and 7,604 achieved at $40 \mathrm{ng} / \mu \mathrm{L}$ and $60 \mathrm{ng} / \mu \mathrm{L}$ of Douglas fir DNA, respectively. 


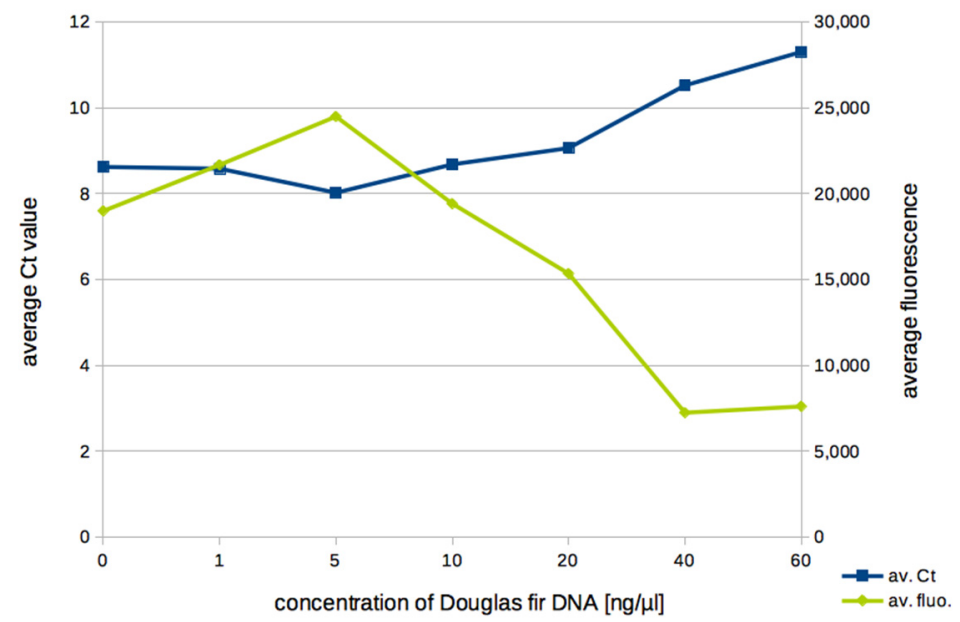

Figure 2. Influence of Douglas fir DNA on the LAMP reaction (GspSSD Isothermal Mastermix; primer set 5-6; qTower ${ }^{3}$ ): Concentration of Douglas fir DNA (x axis) from $0 \mathrm{ng} / \mu \mathrm{L}$ to $60 \mathrm{ng} / \mu \mathrm{L}$; average cycle threshold $(\mathrm{Ct})$ value $(\mathrm{av} . \mathrm{Ct})=$ blue curve; average fluorescence (av. fluo.; averaged values from cycles 40 to 45$)$ = green curve.

A total of 15 different extraction methods were tested and the results of the LAMP reaction evaluated in terms of reaction rate and fluorescence intensity. DNA kits from Omega Bio-tek, Inc., Macherey-Nagel GmbH \& Co. KG, and Qiagen GmbH were characterized by test failures, low yields, and excessively long reaction times, and therefore cannot be recommended for the LAMP reaction. The FTA cards (Whatman) also failed to show any amplification in the LAMP reaction and are therefore unsuitable. Figure 3 shows the LAMP reactions of the best extraction methods in comparison with the synthetic Rhabdocline fragment (no. 1). From an overall perspective, DNA kits from Analytik Jena were found to be the best means of extracting fungal DNA from mixed samples. The innuPREP Stool DNA Kit (Protocol 1: bacterial DNA; Figure 3, no. 3) exhibited slight advantages in terms of yield and the reproducibility of results when compared with the innuPREP Plant DNA Kit protocols tested (Protocol 1: Solution SLS; Figure 3, no. 2). Despite the delay in the start of the reaction, the Plant Material Lysis Kit (OptiGene Ltd.; Figure 3, no. 6 and no. 7) and the Dilution Buffer from the Phire Plant Direct PCR Kit (Fisher Scientific GmbH; Figure 3, no. 4 and no. 5) exhibited very good fluorescence values - and therefore high yields in terms of amplified products - even in the case of sample material without visibly discernible fruiting bodies of $R$. pseudotsugae. 


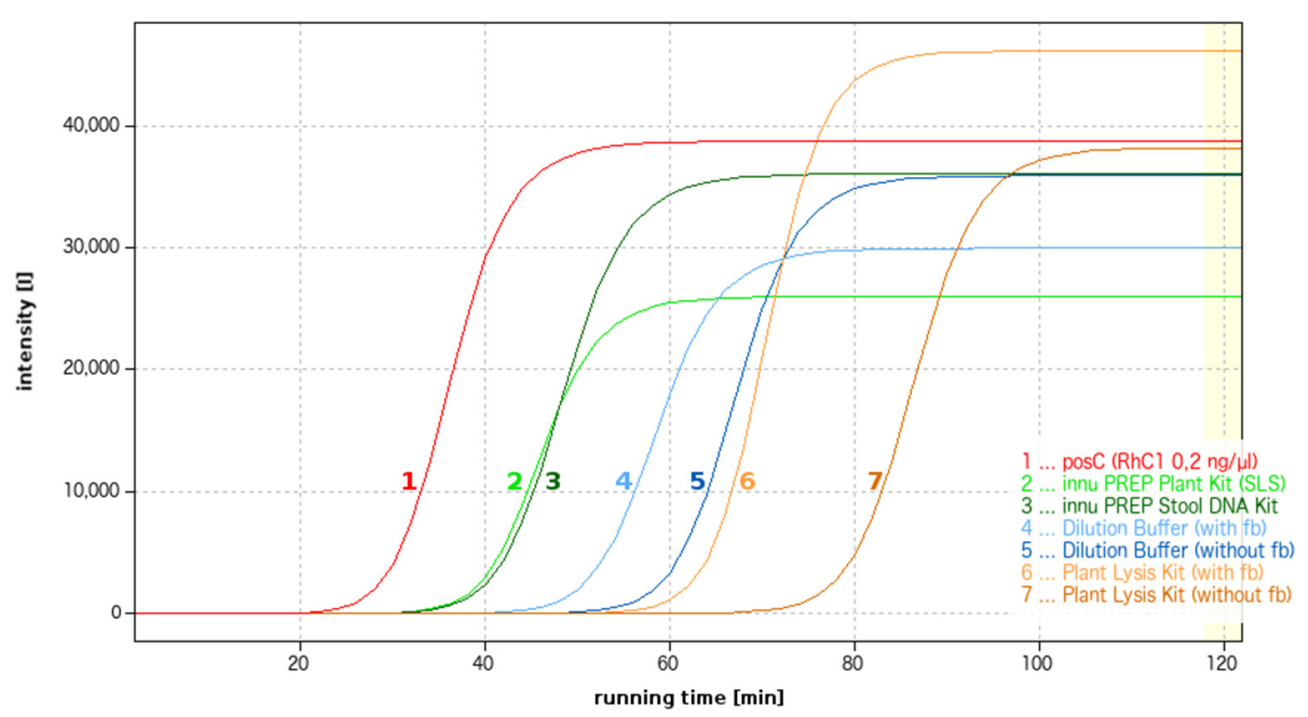

Figure 3. Amplification curves for the qTower $^{3}$ real-time PCR cycler (GspSSD Isothermal Mastermix; primer set 5-6; incubation time: $2 \mathrm{~h}$ ): LAMP reaction comparison between the synthetic Rhabdocline fragment (no. 1 = positive control (posC); RhC1; $0.2 \mathrm{ng} / \mu \mathrm{L}$ ) and various DNA extraction methods (no. 2 = innuPREP Plant DNA Kit; Protocol 1: Solution SLS; no. 3 = innuPREP Stool DNA Kit; Protocol 1: bacterial DNA; no. 4 = Dilution Buffer, Phire Plant Direct PCR Kit, needles with visible fruiting bodies (fb); no. $5=$ Dilution Buffer, Phire Plant Direct PCR Kit, needles without visible fruiting bodies; no. $6=$ Plant Material Lysis Kit, needles with visible fruiting bodies; no. $7=$ Plant Material Lysis Kit, needles without visible fruiting bodies).

\section{Discussion}

As described above, the ongoing TreeLAMP project has led to the successful establishment of a quick, sensitive LAMP assay for the detection of R. pseudotsugae, the pathogen that causes Rhabdocline needle cast. Working with $R$. pseudotsugae is accompanied by special challenges. On the one hand, the fungus is not yet known to feature any distinctive mycelia, with the visual examination of specimens for fruiting bodies therefore the only available means of macroscopic identification. On the other, the successful cultivation of this obligate biotrophic needle parasite has not yet been reported. The continuous availability of fungal material of high purity is nevertheless a fundamental prerequisite for the establishment of a quick test specific to R. pseudotsugae. The study at hand initially used fruiting bodies dissected from infected needles. With the harvesting of fresh fruiting bodies essentially only possible in May, and with fruiting bodies only found on a limited number of Douglas firs in both years of harvesting, the LAMP assay went on to be established using a synthetic Rhabdocline fragment as a positive control. Neither nested PCR using primer pair RPP1/RPP4 [7] nor the newlyestablished LAMP assay identified any differences between the synthetic fragment and samples taken from fruiting bodies of R. pseudotsugae.

The detection limit of the LAMP assay was found to stand at $0.02 \mathrm{pg} / \mu \mathrm{L}$ (reaction time: $1.5 \mathrm{~h}$ ) in the case of pure R. pseudotsugae samples as well as mixed fungal/Douglas fir samples. A comparison between PCR with subsequent fragment length measurement and LAMP amplification found LAMP to detect a $21 \%$ higher infection rate in seedlings. It is nevertheless to be noted that the ITS product used for nested PCR was also used for the LAMP reaction. On the one hand, this was due to the fact that the DNeasy Plant Mini Kit (Qiagen $\mathrm{GmbH}$ ) was still being used to extract DNA from the seedlings and only identified as unsuitable during the subsequent optimization of DNA extraction for the LAMP assay. On the other, extraction rendered the respective plant material unusable, thus preventing the repeated extraction of DNA from the same plant material. The newlyestablished LAMP assay can nevertheless be assumed to be highly sensitive, as the use of 
an identical DNA template for both methods ensured the availability of a comparable amount of $R$. pseudotsugae DNA for the respective reaction.

Published data on the sensitivity of LAMP reactions varies greatly. To give an example, the LAMP kit for H. pseudoalbidus from OptiGene Ltd. (Horsham, UK) is described as achieving a sensitivity of $7 \mathrm{pg}$ of DNA based on a reaction time of $15.5 \mathrm{~min}$ [11]. The LAMP assay for F. graminearum detects up to $2 \mathrm{pg}$ of the fungus in $30 \mathrm{~min}$ [12], while the assays for $X$. fastidiosa, C. platani, and P. ramorum are characterized by a detection limit of $0.02 \mathrm{pg} / \mu \mathrm{L}$ based on a reaction time of $30 \mathrm{~min}$ [15]. By way of contrast, it is possible to reliably detect $65 \mathrm{pg}$ of $B$. cinerea after a reaction time of $\leq 20 \mathrm{~min}$ [13], with $20 \mathrm{pg}$ of $H$. irregulare detectable in under $40 \mathrm{~min}$ [14]. Despite the large number of reasons to which the varying sensitivity of LAMP assays may be attributable, it is the pathogens involved and the DNA regions upon which LAMP primer selection is based that are most likely to have an influence. The use of a synthetic fragment as a positive control for R. pseudotsugae is unlikely to have played a role, as all sensitivity tests performed within the context of the work at hand were also carried out in combination with DNA extracted from fruiting bodies of the fungus as well as mixed DNA samples.

There is nevertheless a fundamental difference between the newly-established LAMP assay for R. pseudotsugae and other assays in terms of reaction rate. In the work at hand, $1.5 \mathrm{~h}$ are required in order to detect $0.02 \mathrm{pg} / \mu \mathrm{l}$ of $R$. pseudotsugae DNA. Target DNA concentration is known to have an influence on reaction rate, but amplification was found to take $60 \mathrm{~min}$ even at a concentration of $20 \mathrm{pg} / \mu \mathrm{L}$. Other LAMP assays can be evaluated after a reaction time of between $15 \mathrm{~min}$ [11] and $40 \mathrm{~min}$ [14]. In the case of the new $R$. pseudotsugae assay, it is possible that the relatively high GC content in the ITS2 region (59\%) - the region in which the binding sites for primer set 5-6 are located-affects the optimum hybridization of the LAMP primers at the start of the reaction in particular, thus resulting in a delay in the start of the exponential amplification of the LAMP products. A reaction time of $1.5 \mathrm{~h}$ is nevertheless acceptable given the high level of sensitivity achieved by the newly-established LAMP assay.

As reaction time increases, so does the risk of non-specific amplification such as that observed in polymerases from New England Biolabs $\mathrm{GmbH}$, in particular in the study at hand. To give an example, this may be caused by a decrease in intact nucleotides and intact polymerases as reaction time increases, which can lead to mishybridization. Nonspecific amplification and false positive results may naturally also be attributable to nonspecific primer binding. Cross-reactions with closely related species are also regularly described in connection with a variety of LAMP assays. Examples include the ability of the LAMP assay for F. graminearum to detect other species of the genus Fusarium from a reaction time of $50 \mathrm{~min}$ and upwards [12], as well as the cross-reactions with $C$. fimbriata and P. lateralis described in Aglietti et al. [15].

The specificity of the newly-established LAMP primers for R. pseudotsugae was tested with the aid of the AMF sample as well as 15 fungal isolates from Douglas fir needles. The AMF sample facilitated the relatively simple, successful testing of the LAMP primers on a range of different fungal sequences. The fungal isolates, on the other hand, were found to enable specific tests for fungi, including the Douglas fir endophyte $R$. parkeri and $N$. gaeumannii, the pathogen that causes Swiss needle cast. Cross-reactions with other pathogenic species of the genus Rhabdocline were not tested for in the study at hand due to the fact that only $R$. pseudotsugae has been found to exist in Europe to date.

The LAMP amplifications initially observed in the case of R. parkeri and P. macrostoma are to be regarded not as an indication that the LAMP primers lack specificity, but rather as evidence of a high level of sensitivity. The LAMP reaction tests all samples according to the principle of double determination. A positive signal was measured in the case of one of four R. parkeri isolates and one of two samples but not reproduced in the repeated test. The positive result in the case of $R$. parkeri must therefore be attributable to either a non-specific reaction or a sample handling error. In the case of $P$. macrostoma, on the other hand, the positive result is suspected to have been caused by the contamination of the 
culture with $R$. pseudotsugae, as the LAMP reaction only led to a negative test for $P$. macrostoma after the repetition of the DNA extraction.

In summary, the newly-established LAMP assay is characterized by a high level of sensitivity, ease of handling, and minimal investment in equipment. In the case of in situ applications, the LAMP assay is nevertheless only practical in combination with a sample preparation and DNA extraction process that is equally quick and simple to perform. A variety of suitable extraction options were tested, with considerable differences observed between the various methods and manufacturers involved. As expected, the sensitivity of the LAMP reaction when used with direct analysis protocols is somewhat limited when compared with that of a conventional DNA kit. Extraction with the aid of a DNA kit is therefore preferable, provided the technical prerequisites are fulfilled. The innuPREP Stool DNA Kit from Analytik Jena can be recommended for the detection of $R$. pseudotsugae in mixed samples. In the case of testing under more minimalistic conditions, the Plant Material Lysis Kit from OptiGene Ltd. is recommended over other options due to its ease of handling.

\section{Conclusions}

The results presented above demonstrate not only the potential offered by molecularlevel genetic methods where pathogen testing is concerned, but also that such methods do not necessarily involve a high level of technical effort and investment. In addition, it was once again possible to show that R. pseudotsugae is detectable in seedlings, and that latently-infected seeds can therefore carry a risk of infection. From the perspective of plant production, distribution, and cultivation in the forestry sector, this represents a substantial economic risk that is almost impossible to estimate at the present time. Only the rigorous implementation of plant hygiene measures and quality-oriented seed selection can keep pathogens at a tolerable level and, in turn, contribute to the long-term preservation of stable forest stands. In our view, it is therefore vital that threshold values are defined for pathogens in seeds and seed harvesting stands, and that seeds and seedlings are systematically tested before the respective plant material is brought onto the market. Suitable early detection methods are already available for a wide range of relevant pathogens.

\section{Patents}

The registration of a patent for the LAMP primers developed within the framework of the TreeLAMP project is under consideration.

Author Contributions: Conceptualization: K.M., J.-U.P., and B.R.; methodology: K.M., J.-U.P., and B.R.; validation: I.H. and P.S.; formal analysis: K.M., J.-U.P., and B.R.; resources: P.S. and I.H.; data curation: J.-U.P.; writing - original draft preparation: K.M.; writing - review and editing: D.K., I.H., J.-U.P., B.R., and P.S.; visualization: K.M.; supervision: D.K.; project administration: I.H. All authors have read and agreed to the published version of the manuscript.

Funding: This research was funded by Federal Ministry of Food and Agriculture; Fachagentur Nachwachsende Rohstoffe e. V. (grant number 22026416).

Institutional Review Board Statement: Not applicable.

Informed Consent Statement: Not applicable.

Data Availability Statement: The data presented in this study are available on request from the corresponding author. A patent application for the developed LAMP primers is currently being considered. Publication of primer sequences is therefore not possible.

Acknowledgments: We thank state forest administrations for the supply of experimental plots and plant material. We also thank Julianne Tippmann and Isa Szut (Institute of Forest Botany and Forest Zoology, Technische Universität Dresden) for their valuable support in the laboratory. 
Conflicts of Interest: The authors declare no conflicts of interest. The funders had no role in the design of the study; in the collection, analyses, or interpretation of data; in the writing of the manuscript; or in the decision to publish the results.

\section{References}

1. Barnes, I.; Kirisits, T.; Wingfield, M.J.; Wingfield, B.D. Needle blight of pine caused by two species of Dothistroma in Hungary. For. Pathol. 2011, 41, 361-369, doi:10.1111/j.1439-0329.2010.00689.x.

2. Ioos, R.; Fabre, B.; Saurat, C.; Fourrier, C.; Frey, P.; Marçais, B. Development, Comparison, and Validation of Real-Time and Conventional PCR Tools for the Detection of the Fungal Pathogens Causing Brown Spot and Red Band Needle Blights of Pine. Phytopathology 2010, 100, 105-114, doi:10.1094/PHYTO-100-1-0105.

3. Schweigkofler, W.; O'Donnell, K.; Garbelotto, M. Detection and Quantification of Airborne Conidia of Fusarium circinatum, the Causal Agent of Pine Pitch Canker, from Two California Sites by Using a Real-Time PCR Approach Combined with a Simple Spore Trapping Method. Appl. Env. . Microbiol. 2004, 70, 3512-3520, doi:10.1128/AEM.70.6.3512-3520.2004.

4. Luchi, N.; Pepori, A.L.; Bartolini, P.; Ioos, R.; Santini, A. Duplex real-time PCR assay for the simultaneous detection of Caliciopsis pinea and Fusarium circinatum in pine samples. Appl. Microbiol. Biotechnol. 2018, 102, 7135-7146, doi:10.1007/s00253018-9184-1.

5. Ioos, R.; Laugustin, L.; Schenck, N.; Rose, S.; Husson, C.; Frey, P. Usefulness of single copy genes containing introns in Phytophthora for the development of detection tools for the regulated species P. ramorum and P. fragariae. Eur. J. Plant Pathol. 2006, 116, 171-176, doi:10.1007/s10658-006-9051-2.

6. Schena, L.; Duncan, J.M.; Cooke, D.E.L. Development and application of a PCR-based 'molecular tool box' for the identification of Phytophthora species damaging forests and natural ecosystems. Plant Pathol. 2008, 57, 64-75, doi:10.1111/j.13653059.2007.01689.x.

7. Catal, M. Development and testing of oligonucleotide probes for detection and identification of some fungal pathogens and endophytes of conifers. Ph.D. Thesis, Department of Plant Pathology, Michigan State University, East Lansing, MI, USA, 2002.

8. Smith, D.R.; Stanosz, G.R. A Species-Specific PCR Assay for Detection of Diplodia pinea and D. scrobiculata in Dead Red and Jack Pines with Collar Rot Symptoms. Plant Dis. 2006, 90, 307-313, doi:10.1094/PD-90-0307.

9. Johansson, S.B.K.; Vasaitis, R.; Ihrmark, K.; Barklund, P.; Stenlid, J. Detection of Chalara fraxinea from tissue of Fraxinus excelsior using species-specific ITS primers. For. Pathol. 2010, 40, 111-115, doi:10.1111/j.1439-0329.2009.00614.x.

10. Notomi, T.; Okayama, H.; Masubuchi, H.; Yonekawa, T.; Watanabe, K.; Amino, N.; Hase, T. Loop-mediated isothermal amplification of DNA. Nucl. Acids Res. 2000, 28, e63, doi:10.1093/nar/28.12.e63.

11. Harrison, C.; Tomlinson, J.; Ostoja-Starzewska, S.; Boonham, N. Evaluation and validation of a loop-mediated isothermal amplification test kit for detection of Hymenoscyphus fraxineus. Eur. J. Plant Pathol. 2017, 149, 253-259, doi:10.1007/s10658-017-1179-8.

12. Niessen, L.; Vogel, R.F. Detection of Fusarium graminearum DNA using a loop-mediated isothermal amplification (LAMP) assay. Int. J. Food Microbiol. 2010, 140, 183-191, doi:10.1016/j.ijfoodmicro.2010.03.036.

13. Tomlinson, J.A.; Dickinson, M.J.; Boonham, N. Detection of Botrytis cinerea by loop-mediated isothermal amplification. Lett. Appl. Microbiol. 2010, 51, 650-657, doi:10.1111/j.1472-765X.2010.02949.x.

14. Sillo, F.; Giordano, L.; Gonthier, P. Fast and specific detection of the invasive forest pathogen Heterobasidion irregulare through a Loop-mediated isothermal AMPlification (LAMP) assay. For. Pathol. 2018, 48, e12396, doi:10.1111/efp.12396.

15. Aglietti, C.; Luchi, N.; Pepori, A.L.; Bartolini, P.; Pecori, F.; Raio, A.; Capretti, P.; Santini, A. Real-time loop-mediated isothermal amplification: an early-warning tool for quarantine plant pathogen detection. AMB Express 2019, 9, doi:10.1186/s13568-019-0774-9.

16. Menzel, P.; Ng, K.L.; Krogh, A. Fast and sensitive taxonomic classification for metagenomics with Kaiju. Nat. Commun. 2016, 7 , 11257, doi:10.1038/ncomms11257.

17. White, T.J.; Bruns, T.; Lee, S.; Taylor, J. Amplification and direct sequencing of fungal ribosomal RNA genes for phylogenetics. In PCR Protocols: A Guide to Methods and Applications; Innis, M.A., Gelfand, D.H., Sninsky, J.J., White, T.J., Eds.; Academic Press Inc.: New York, NY, USA, 1990; Volume 18, pp. 315-322, ISBN 0-12-372181-4.

18. Gardes, M.; Bruns, T.D. ITS primers with enhanced specificity for basidiomycetes - application to the identification of mycorrhizae and rusts. Mol. Ecol. 1993, 2, 113-118, doi:10.1111/j.1365-294X.1993.tb00005.x.

19. Morgenstern, K.; Döring, M.; Krabel, D. Rhabdocline needle cast-investigations on various Douglas fir tissue types. Eur. J. Plant Pathol. 2013, 137, 495-504, doi:10.1007/s10658-013-0261-0. 